\title{
ESTIMATING MULTIVARIATE EXTREMAL DEPENDENCE: A NEW PROPOSAL
}

\author{
M. FERREIRA
}

\begin{abstract}
Multivariate extreme values require the use of extreme-value copulas, as they appear in the limit of componentwise maxima. These can be characterized by the so-called Pickands dependence function. A new multivariate nonparametric estimator will be presented, along with convergence properties. Based on simulations, we will analyze its performance and compare with well-known estimators from the literature.
\end{abstract}

\section{INTRODUCTION}

The use of copulas allows us to focus only on the dependence characterizing the margins of a random vector. Extreme value copulas define the dependence structure of random vectors coming from a multivariate extreme-values model.

Let $\left(X_{1}, \ldots, X_{d}\right)$ be a random vector with continuous distribution function (df) $F$ and marginal df's $F_{j}$, such that

$$
F\left(x_{1}, \ldots, x_{d}\right)=C\left(F_{1}\left(x_{1}\right), \ldots, F_{d}\left(x_{d}\right)\right),
$$

i.e., copula $C$ is the df of $\left(F_{1}\left(X_{1}\right), \ldots, F_{d}\left(X_{d}\right)\right)$.

An extreme-value copula $C$ can be expressed in terms of tail dependence measures. For instance, the stable tail dependence function $\ell:[0, \infty)^{d} \rightarrow[0, \infty)$, given by

$$
\ell\left(x_{1}, \ldots, x_{d}\right)=\lim _{t \rightarrow \infty} t \mathrm{P}\left(\bigcup_{j=1}^{d}\left\{F_{j}\left(X_{j}\right)>1-x_{j} / t\right\}\right)
$$

is such that

$$
C\left(u_{1}, \ldots, u_{d}\right)=\exp \left(-\ell\left(-\log u_{1}, \ldots,-\log u_{d}\right)\right), \quad\left(u_{1}, \ldots, u_{d}\right) \in(0,1]^{d},
$$

and satisfies the properties of homogeneity of order one (i.e.,

$$
\ell\left(\left(s x_{1}, \ldots, s x_{d}\right)\right)=s \ell\left(x_{1}, \ldots, x_{d}\right),
$$

$\forall s>0)$, convexity, $\ell\left(\mathbf{e}_{j}\right)=1$ for $j=1, \ldots, d\left(\mathbf{e}_{j}\right.$ is the $j$ th unit vector in $\left.\mathbb{R}^{d}\right)$, as well as, it is lower and upper bounded by $\max \left(x_{1}, \ldots, x_{d}\right)$ and $x_{1}+\cdots+x_{d}$, respectively. We can also state $C$ as

$$
C\left(u_{1}, \ldots, u_{d}\right)=\exp \left(\left(\sum_{j=1}^{d} \log u_{j}\right) A\left(\frac{\log u_{1}}{\sum_{j=1}^{d} \log u_{j}}, \ldots, \frac{\log u_{d-1}}{\sum_{j=1}^{d} \log u_{j}}\right)\right),
$$

2010 Mathematics Subject Classification. Primary 62G05; Secondary 62G32.

Key words and phrases. Extreme value copula, multivariate Pickands dependence function, nonparametric estimation. 
$\left(u_{1}, \ldots, u_{d}\right) \in(0,1]^{d} \backslash\{(1, \ldots, 1)\}$, where $A$ is the Pickands dependence function, related with function $\ell$ via

$$
A\left(w_{1}, \ldots, w_{d-1}\right) \equiv A\left(\frac{x_{1}}{\sum_{j=1}^{d} x_{j}}, \ldots, \frac{x_{d-1}}{\sum_{j=1}^{d} x_{j}}\right)=\frac{\ell\left(x_{1}, \ldots, x_{d}\right)}{\sum_{j=1}^{d} x_{j}} .
$$

The Pickands dependence function $A: \mathcal{S}_{d-1} \rightarrow[1 / d, 1]$ corresponds to the restriction of $\ell$ to the unit simplex $\mathcal{S}_{d-1}=\left\{\left(w_{1}, \ldots, w_{d}\right) \in[0,1]^{d}: \sum_{j=1}^{d} w_{j}=1\right\}$, being also convex and such that $A\left(\mathbf{e}_{j}\right)=1$ for $j=1, \ldots, d$, and having bounds $\max \left(w_{1}, \ldots, w_{d}\right) \leq A(\mathbf{w}) \leq 1$, $\forall \mathbf{w} \in \mathcal{S}_{d-1}$.

Therefore, inference on extreme-value copulas resumes to the estimation of a tail dependence function. The main approaches to this topic in literature are based on the method of moments of an exponential distribution or a logarithm of this latter, with parameter $A(t)$. The first leads to the Pickands estimator (Pickands [13]) and variants of it (Deheuvels [5]; Hall and Tajvidi [12]), while the second leads to the CapéraàFougères-Genest (CFG) estimator (Capéraà et al. 4]). Extensions to general dimensions can be found in Zhang et al. ([18]) and Gudendorf and Segers ([10]). The more plausible assumption of unknown margins was treated in Abdous and Ghoudi ([1]), Genest and Segers ([9]), Gudendorf and Segers ([1]); see also the references therein. A new class of minimum distance estimators was proposed in Bücher et al. ([3]) in a bivariate framework and estimators based on projections on Hilbert space $L^{2}\left(\mathcal{S}_{d-1}\right)$ of real-valued and Lebesgue square-integrable functions can be seen in Fils-Villetard et al. ([] $)$ for $d=2$ and Gudendorf and Segers ([1] ) for arbitrary dimensions.

In this work we address the nonparametric estimation of $A$ in arbitrary dimensions, by presenting a new estimator in the same spirit of Pickands and CFG estimators, that is, based on the method of moments. This extends the bivariate approach presented in Ferreira $([7])$, as will be seen in Section 2, Asymptotic properties are simple adaptations of the results in Gudendorf and Segers ([1] $)$. In Section 3 we analyze the finite-sample performance of the new proposal through simulation, in comparison with estimators well known in the literature. A short discussion is presented in Section 4 .

\section{Estimation of Pickands DePendence Function}

Consider a sequence $\left\{\mathbf{X}_{n}\right\}_{n \geq 1}$ of independent $d$-variate random vectors with multivariate common df $F$ having continuous marginal df's $F_{j}, j=1, \ldots, d$. Assuming known margins, the empirical copula is written as

$$
C_{n}(\mathbf{u})=\frac{1}{n} \sum_{i=1}^{n} \mathbb{1}\left(U_{i, 1} \leq u_{1}, \ldots, U_{i, d} \leq u_{d}\right), \quad \mathbf{u}=\left(u_{1}, \ldots, u_{d}\right) \in[0,1]^{d},
$$

with $U_{i, j}=F_{j}\left(X_{i, j}\right), i=1, \ldots, n$ and $j=1, \ldots, d$, where $\mathbb{1}$ is the indicator function. By classical theory of empirical processes, we have

$$
\mathbb{C}_{n}=\sqrt{n}\left(C_{n}-C\right) \rightarrow \zeta, \quad n \rightarrow \infty,
$$

weakly in the space $l^{\infty}\left([0,1]^{d}\right)$ of real-valued bounded functions on $[0,1]^{d}$ equipped with the topology of uniform convergence, where $\zeta$ is a centered Gaussian process with continuous trajectories and covariance function

$$
\operatorname{cov}(\zeta(\mathbf{u}), \zeta(\mathbf{v}))=C(\mathbf{u} \wedge \mathbf{v})-C(\mathbf{u}) C(\mathbf{v}), \quad \mathbf{u}, \mathbf{v} \in[0,1]^{d},
$$

where the operator ' $\wedge$ ' denotes the 'minimum'. The weak convergence is in the sense of Hoffman-Jørgensen as in van der Vaart and Wellner ([17, Section 1.5). More precisely, 
a set function $\mu$ is a finite nonnegative monotone supadditive set function (FNNMSA) on a topological space $E$ if it is defined on the set of all open sets in $E$,

$$
0 \leq \mu(G) \leq \mu(Q) \leq \mu(E)<\infty
$$

if $G \subset Q$ are open sets and $\mu(G)+\mu(Q) \leq \mu(G \cup Q)$ for each couple of disjoint open sets $G, Q$. Let $\left\{\mu_{n}\right\}$ be a sequence of FNNMSA's on $E, \mu$ be a FNNMSA on $E$ and $S$ a Borel subset of $E$. We say that $\mu_{n}$ converges weakly to $\mu$ in $(S, E)$ in the sense of Hoffman-Jørgensen, if $\liminf _{n} \mu_{n}(G) \geq \mu(G)$ holds for each open set $G$ and

$$
\lim _{n} \mu_{n}(G)=\mu(G)=\mu(E)
$$

whenever $G \supset S$.

Under the more realistic assumption of unknown margins, and in a full nonparametric framework, we can use empirical df estimation as

$$
F_{n, j}(x)=\frac{1}{n+1} \sum_{i=1}^{n} \mathbb{1}\left(X_{i, j} \leq x\right), \quad j=1, \ldots, d,
$$

where denominator $n+1$ instead of $n$ concerns accuracy (see Beirlant et al. [2]; Section 9.4.1). Now consider $\widehat{U}_{i, j}=F_{n, j}\left(X_{i, j}\right)$ and denote by $\widehat{C}_{n}$ the empirical copula obtained from (11) by replacing $U_{i, j}$ by $\widehat{U}_{i, j}, i=1, \ldots, n$ and $j=1, \ldots, d$, as well as, the respective process $\widehat{\mathbb{C}}_{n}=\sqrt{n}\left(\widehat{C}_{n}-C\right)$. Assume also that the condition below holds:

(A) The first-order partial derivatives $\dot{C}_{j}, j=1, \ldots, d$, exist and are continuous on the respective set $A_{j}=\left\{\mathbf{u} \in[0,1]^{d}: 0<u_{j}<1\right\}$, as well as, the second-order partial derivatives $\ddot{C}_{l, j}, l, j=1, \ldots, d$ exist and are continuous on $A_{l} \cap A_{j}$ ( $l$ and $j$ not necessarily distinct), and such that

$$
\sup _{\mathbf{u} \in A_{l} \cap A_{j}} \max \left\{u_{l}\left(1-u_{l}\right), u_{j}\left(1-u_{j}\right)\right\}\left|\ddot{C}_{l, j}(\mathbf{u})\right|<\infty .
$$

Then, the processes $\widehat{\mathbb{C}}_{n}$ and $\mathbb{C}_{n}$ are related through

$$
\widehat{\mathbb{C}}_{n}(\mathbf{u})=\mathbb{C}_{n}(\mathbf{u})-\sum_{j=1}^{d} \dot{C}_{j}(\mathbf{u}) \mathbb{C}_{n, j}\left(u_{j}\right)+R_{n}(\mathbf{u}),
$$

with $\mathbb{C}_{n, j}\left(u_{j}\right)=\mathbb{C}_{n}\left(1, \ldots, 1, u_{j}, 1, \ldots, 1\right), j=1, \ldots, d$, and such that

$$
\sup _{\mathbf{u} \in[0,1]^{d}}\left|R_{n}(\mathbf{u})\right| O\left((\log n)^{1 / 2}((\log \log n) / n)^{1 / 4}\right) \quad \text { almost surely. }
$$

Therefore, it follows that $\widehat{\mathbb{C}}_{n} \rightarrow \mathbb{C}$ weakly in space $l^{\infty}\left([0,1]^{d}\right)$ equipped with the topology of uniform convergence, where

$$
\mathbb{C}(\mathbf{u})=\zeta(\mathbf{u})-\sum_{j=1}^{d} \dot{C}_{j}(\mathbf{u}) \zeta_{j}\left(u_{j}\right),
$$

with $\zeta_{j}\left(u_{j}\right)=\zeta\left(1, \ldots, 1, u_{j}, 1, \ldots, 1\right), j=1, \ldots, d$. See Segers $([15])$ and the references therein.

In the bivariate case $(d=2)$, if we take

$$
\xi(t)=\frac{-\log U_{1}}{1-t} \wedge \frac{-\log U_{2}}{t}, \quad 0<t<1,
$$

with $\xi(0)=-\log U_{1}$ and $\xi(1)=-\log U_{2}$, we have $\xi(t)$ exponentially distributed with mean values

$$
\mathrm{E}(\xi(t))=1 / A(t) \quad \text { and } \quad \mathrm{E}(\log (\xi(t)))=-\log (A(t))-\gamma
$$


where $\gamma$ denotes Euler's constant $\int_{0}^{\infty} \log (x) e^{-x} d x \approx 0.577$. These relations are the bases of, respectively, the Pickands and the CFG estimators by considering a kind of method of moments, that is, we have

$$
1 / \widehat{A}_{n}^{P}(t)=\frac{1}{n} \sum_{i=1}^{n} \widehat{\xi}_{i}(t) \quad \text { and } \quad \log \left(\widehat{A}_{n}^{C F G}(t)\right)=-\gamma-\frac{1}{n} \sum_{i=1}^{n} \log \left(\widehat{\xi}_{i}(t)\right)
$$

with

$$
\widehat{\xi}_{i}(t)=\frac{-\log \widehat{U}_{i, 1}}{1-t} \wedge \frac{-\log \widehat{U}_{i, 2}}{t}, \quad 0<t<1 .
$$

In arbitrary dimensions $d$, formulas above become, for $\mathbf{w} \in \mathcal{S}_{d-1}$,

$$
1 / \widehat{A}_{n}^{P}(\mathbf{w})=\frac{1}{n} \sum_{i=1}^{n} \widehat{\xi}_{i}(\mathbf{w})
$$

and

with

$$
\log \left(\widehat{A}_{n}^{C F G}(\mathbf{w})\right)=-\gamma-\frac{1}{n} \sum_{i=1}^{n} \log \left(\widehat{\xi}_{i}(\mathbf{w})\right)
$$

$$
\widehat{\xi}_{i}(\mathbf{w})=\bigwedge_{j=1}^{d} \frac{-\log \widehat{U}_{i, j}}{w_{j}} .
$$

In order to satisfy the endpoint constraints $A\left(\mathbf{e}_{j}\right)=1$, where $\mathbf{e}_{j}$ corresponds to the $j$ th standard unit vector in $\mathbb{R}^{d}, j=1, \ldots, d$, the endpoint corrected versions

$$
1 / \widehat{A}_{n, c}^{P}(\mathbf{w})=\frac{1}{\widehat{A}_{n}^{P}(\mathbf{w})}-\sum_{j=1}^{d} \lambda_{j}(\mathbf{w})\left(\frac{1}{\widehat{A}_{n}^{P}\left(\mathbf{e}_{j}\right)}-1\right)
$$

and

$$
\log \left(\widehat{A}_{n, c}^{C F G}(\mathbf{w})\right)=\log \widehat{A}_{n}^{C F G}(\mathbf{w})-\sum_{j=1}^{d} \lambda_{j}(\mathbf{w}) \log \widehat{A}_{n}^{C F G}\left(\mathbf{e}_{j}\right)
$$

are considered, where each function $\lambda_{j}: \mathcal{S}_{d-1} \rightarrow \mathbb{R}$ is continuous and satisfies $\lambda_{j}\left(\mathbf{e}_{l}\right)=\delta_{j l}$ (Kronecker delta) for $j, l \in\{1, \ldots, d\}$. A common choice is $\lambda_{j}(\mathbf{w})=w_{j}$. For large sample sizes, the endpoint corrections are negligible since

$$
1 / \widehat{A}_{n}^{P}\left(\mathbf{e}_{j}\right)=1+O(\log n / n) \text { and } \log \widehat{A}_{n}^{C F G}\left(\mathbf{e}_{j}\right)=O\left((\log n)^{2} / n\right)
$$

(see Gudendorf and Segers [10]). Another correction of the Pickands estimator based on Hall and Tajvidi [12] extends to

$$
\widehat{A}_{n}^{H T}(\mathbf{w})=\frac{\widehat{A}_{n}^{P}(\mathbf{w})}{\widehat{A}_{n}^{P}\left(\mathbf{e}_{j}\right)}=\widehat{A}_{n}^{P}(\mathbf{w})(1+O((\log n) / n)),
$$

and thus $\widehat{A}_{n}^{H T}(\mathbf{w})$ and $\widehat{A}_{n}^{P}(\mathbf{w})$ are asymptotically equivalent.

Under condition (A), Gudendorf and Segers [10] derive the weak limit processes

$$
-A^{2}(\mathbf{w}) \int_{0}^{1} \mathbb{C}\left(u^{w_{1}}, \ldots, u^{w_{d}}\right) d u / u
$$

and

$$
A(\mathbf{w}) \int_{0}^{1} \mathbb{C}\left(u^{w_{1}}, \ldots, u^{w_{d}}\right) d u /(u \log u)
$$

of, respectively, $\mathbb{A}^{P}=\sqrt{n}\left(\widehat{A}_{n}^{P}-A\right)$ and $\mathbb{A}^{C F G}=\sqrt{n}\left(\widehat{A}_{n}^{C F G}-A\right)$, as $n \rightarrow \infty$, in the space $\mathcal{C}\left(\mathcal{S}_{d-1}\right)$ of continuous real-valued functions endowed with the uniform convergence topology. 
In Ferreira [7, a new estimator for the bivariate Pickands dependence function was considered which also departs from a functional relation with a first-order moment as in Pickands and CFG methodology. More precisely, taking

$$
\eta(t)=U_{1}^{1 /(1-t)} \vee U_{2}^{1 / t}
$$

with $\eta(0)=U_{1}$ and $\eta(1)=U_{2}$, we have (Ferreira and Ferreira, [6]; Proposition 3.1)

$$
E(\eta(t))=1-\frac{1}{1+A(t)} .
$$

An analogous reasoning, as in Pickands and CFG estimators, leads to

$$
1-1 /\left(1+\widehat{A}_{n}^{F F}(t)\right)=\frac{1}{n} \sum_{i=1}^{n} \widehat{\eta}_{i}(t),
$$

(the 'FF' notation stands for the above cited work) where

$$
\widehat{\eta}_{i}(t)=\widehat{U}_{i, 1}^{1 /(1-t)} \vee \widehat{U}_{i, 2}^{1 / t}, \quad 0<t<1 .
$$

Extending to a general dimension $d$, if

$$
\widehat{\eta}_{i}(\mathbf{w})=\bigvee_{j=1}^{d} \widehat{U}_{i, j}^{1 / w_{j}}
$$

for $\mathbf{w} \in \mathcal{S}_{d-1}$, we state the FF estimator as

$$
1-\frac{1}{1+\widehat{A}_{n}^{F F}(\mathbf{w})}=\frac{1}{n} \sum_{i=1}^{n} \widehat{\eta}_{i}(\mathbf{w}) .
$$

Observe that it already satisfies the constraints $\widehat{A}_{n}^{F F}\left(\mathbf{e}_{j}\right)=1, j=1, \ldots, d$, since

$$
\frac{1}{n} \sum_{i=1}^{n} \widehat{\eta}_{i}\left(\mathbf{e}_{j}\right)=\frac{1}{n} \sum_{i=1}^{n} \widehat{U}_{i, j}=\frac{1}{n} \sum_{i=1}^{n} \frac{i}{n+1}=\frac{1}{2} .
$$

The asymptotic properties of FF estimator are simple adaptations of the results above concerning Pickands and CFG estimators.

Proposition 2.1. Under the assumption in (A), we have

$$
\sqrt{n}\left(\widehat{A}_{n}^{F F}(\mathbf{w})-A(\mathbf{w})\right) \rightarrow-(A(\mathbf{w})+1)^{2} \int_{0}^{1} \mathbb{C}\left(u^{w_{1}}, \ldots, u^{w_{d}}\right) d u,
$$

weakly in $\mathcal{C}\left(\mathcal{S}_{d-1}\right)$ equipped with the topology of the uniform convergence.

Proof. Observe that, for $\mathbf{w} \in \mathcal{S}_{d-1}$,

$$
\begin{aligned}
1-\frac{1}{1+\widehat{A}_{n}^{F F}(\mathbf{w})} & =\frac{1}{n} \sum_{i=1}^{n} \int_{0}^{1} \mathbb{1}\left(\widehat{\eta}_{i}(\mathbf{w}) \leq u\right) d u \\
& =\frac{1}{n} \sum_{i=1}^{n} \int_{0}^{1} \mathbb{1}\left(\widehat{U}_{i, 1} \leq u^{w_{1}}, \ldots, \widehat{U}_{i, j} \leq u^{w_{j}}\right) d u \\
& =\int_{0}^{1} \widehat{C}_{n}\left(u^{w_{1}}, \ldots, u^{w_{j}}\right) d u .
\end{aligned}
$$

Changing the variable $u=e^{-s}$ in the integral above, we have

$$
\sqrt{n}\left(\frac{1}{1+\widehat{A}_{n}^{F F}(\mathbf{w})}-\frac{1}{1+A(\mathbf{w})}\right)=\int_{0}^{\infty} \mathbb{C}_{n}\left(e^{-s w_{1}}, \ldots, e^{-s w_{j}}\right) h(s) d s,
$$


TABLE 1. Empirical MISE values obtained for estimators CFG, P, HT and FF of the Pickands dependence function.

\begin{tabular}{l|cccc} 
& & & & \\
$n=1000$ & CFG & P & HT & FF \\
\hline \hline $\log (0.5)$ & $0.7192 \times 10^{-4}$ & $0.7181 \times 10^{-4}$ & $0.7378 \times 10^{-4}$ & $0.7995 \times 10^{-4}$ \\
$\log (0.01)$ & $0.1103 \times 10^{-7}$ & $6.9359 \times 10^{-7}$ & $0.0090 \times 10^{-7}$ & $1.1913 \times 10^{-7}$ \\
$\log (0.99)$ & $0.1557 \times 10^{-3}$ & $0.2976 \times 10^{-3}$ & $0.2998 \times 10^{-3}$ & $0.1805 \times 10^{-3}$ \\
$\operatorname{alog}(0.5)$ & $0.1955 \times 10^{-3}$ & $0.2322 \times 10^{-3}$ & $0.2351 \times 10^{-3}$ & $0.1815 \times 10^{-3}$ \\
$\operatorname{alog}(0.01)$ & $0.1950 \times 10^{-3}$ & $0.1894 \times 10^{-3}$ & $0.1907 \times 10^{-3}$ & $0.1861 \times 10^{-3}$ \\
$\operatorname{alog}(0.99)$ & $0.2377 \times 10^{-3}$ & $0.3603 \times 10^{-3}$ & $0.3629 \times 10^{-3}$ & $0.2354 \times 10^{-3}$ \\
\hline & & & & \\
$n=100$ & $\mathrm{CFG}$ & $\mathrm{P}$ & $\mathrm{HT}$ & $\mathrm{FF}$ \\
\hline \hline $\log (0.5)$ & $0.6144 \times 10^{-3}$ & $0.6379 \times 10^{-3}$ & $0.6721 \times 10^{-3}$ & $0.7778 \times 10^{-3}$ \\
$\log (0.01)$ & $0.0029 \times 10^{-5}$ & $3.0273 \times 10^{-5}$ & $0.0024 \times 10^{-5}$ & $1.1742 \times 10^{-5}$ \\
$\log (0.99)$ & $0.3840 \times 10^{-2}$ & $0.4487 \times 10^{-2}$ & $0.4681 \times 10^{-2}$ & $0.2921 \times 10^{-2}$ \\
$\operatorname{alog}(0.5)$ & $0.1919 \times 10^{-2}$ & $0.1981 \times 10^{-2}$ & $0.2090 \times 10^{-2}$ & $0.1997 \times 10^{-2}$ \\
$\operatorname{alog}(0.01)$ & $0.1510 \times 10^{-2}$ & $0.1612 \times 10^{-2}$ & $0.1711 \times 10^{-2}$ & $0.1531 \times 10^{-2}$ \\
$\operatorname{alog}(0.99)$ & $0.3306 \times 10^{-2}$ & $0.3674 \times 10^{-2}$ & $0.3837 \times 10^{-2}$ & $0.2351 \times 10^{-2}$
\end{tabular}

with $h(s)=e^{-s}$. The rest of the proof is depicted from the relative to Theorem 1 in Gudendorf and Segers [11.

\section{Simulation STUdy}

In order to assess the finite-sample behavior of $\mathrm{FF}$ estimator in comparison with the corrected versions of Pickands, CFG and Hall and Tajvidi estimators, we conduct a simulation study based on Monte-Carlo approximations for the mean integrated squared error $\left(M I S E=\mathrm{E}\left(\int_{0}^{1}\left(\widehat{A}_{n}-A\right)^{2}\right)\right)$. We have considered the case of dimension $d=3$ and generated 100 replicas of size $n=100,1000$ of the symmetric logistic dependence

$$
\ell\left(x_{1}, x_{2}, x_{3}\right)=\left(x_{1}^{1 / \alpha}+x_{2}^{1 / \alpha}+x_{3}^{1 / \alpha}\right)^{\alpha}
$$

and asymmetric logistic

$$
\ell\left(x_{1}, x_{2}, x_{3}\right)=\sum_{J \in \mathcal{D}}\left(\sum_{j \in J}\left(\psi_{J, j} x_{j}\right)^{1 / \alpha_{J}}\right)^{\alpha_{J}},
$$

where $\mathcal{D}$ is the set of nonempty subsets of $D=\{1,2,3\}, 0<\alpha, \alpha_{J} \leq 1$ are the dependence parameters and $\psi_{J, j} \geq 0$ are the asymmetry parameters such that $\sum_{J \ni j} \psi_{J, j}=1$, for $j \in D$ and $J \in \mathcal{D}$ (Tawn [16]).

We used the dependence $0.01,0.5$ and 0.99 in both models and the same asymmetry was assumed in each case of the asymmetric model, with $\psi_{\{1\}, 1}=0.4, \psi_{\{2\}, 2}=0$, $\psi_{\{3\}, 3}=0.6, \psi_{\{1,2\}, 1}=0.3, \psi_{\{1,2\}, 2}=0.2, \psi_{\{1,3\}, 1}=0.1, \psi_{\{1,3\}, 3}=0.1, \psi_{\{2,3\}, 2}=0.4$, $\psi_{\{2,3\}, 3}=0.1, \psi_{\{1,2,3\}, 1}=0.2, \psi_{\{1,2,3\}, 2}=0.4$ and $\psi_{\{1,2,3\}, 3}=0.2$. The results are reported in Table 1. The FF estimator behaves particularly well in the case of (almost) independence (dependence parameter set at 0.99), the most critical case in the context of tail dependence inference. It also presents the best performance in the asymmetric logistic model for large sample sizes $(n=1000)$. Under dependence $(\alpha \leq 0.5)$ within the symmetric model, the best choice relies on the CFG estimator. 


\section{Discussion}

The Pickands dependence function is a crucial structure in what concerns multivariate extreme values inference. The new methodology presented here is revealed to be simple and has promising results in this framework. However, similar to the well-known CFG and Pickands estimators, the requisites of a Pickands dependence function may not always be met by the estimates. In a future work, we intend to pursue further developments, namely, the construction of estimators that satisfy the requirements beyond the endpoint restrictions, for example, following a similar approach of Gudendorf and Segers [1].

\section{BIBLIOGRAPHY}

1. B. Abdous and K. Ghoudi, Non-parametric estimators of multivariate extreme dependence functions, J. Nonparametr. Statist. 17 (2005), 915-935. MR2192166

2. J. Beirlant, Y. Goegebeur, J. Segers, and J. Teugels, Statistics of Extremes: Theory and Applications, Wiley, Chichester, 2004. MR2108013

3. A. Bücher, H. Dette, and S. Volgushev, New estimators of the Pickands dependence function and a test for extreme-value dependence, Ann. Statist. 39 (2011), no. 4, 1963-2006. MR.2893858

4. P. Capéraà, A.-L. Fougères, and C. Genest, A nonparametric estimation procedure for bivariate extreme value copulas, Biometrika 84 (1997), 567-577.

5. P. Deheuvels, On the limiting behavior of the Pickands estimator for bivariate extreme value distributions, Statist. Probab. Lett. 12 (1991), 429-439. MR1142097

6. H. Ferreira and M. Ferreira, On extremal dependence of block vectors, Kybernetika 48 (2012), no. 5, 988-1006. MR3086865

7. M. Ferreira, A new estimator for the Pickands dependence function (2015). (submitted)

8. A. Fils-Villetard, A. Guillou, and J. Segers, Projection estimators of Pickands dependence functions, Canad. J. Statist. 36 (2008), 369-382. MR2456011

9. C. Genest and J. Segers, Rank-based inference for bivariate extreme-value copulas, Ann. Statist. 37 (2009), no. 5B, 2990-3022. MR2541453

10. G. Gudendorf and J. Segers, Nonparametric estimation of an extreme-value copula in arbitrary dimensions, J. Multivariate Anal. 102 (2011), no. 1, 37-47. MR2729418

11. G. Gudendorf and J. Segers, Nonparametric estimation of multivariate extreme-value copulas, J. Statist. Plann. Inference 142 (2012), 3073-3085. MR2956794

12. P. Hall and N. Tajvidi, Distribution and dependence-function estimation for bivariate extremevalue distributions, Bernoulli 6 (2000), 835-844. MR.1791904

13. J. Pickands, Multivariate extreme value distributions (with a discussion), Proceedings of the 43rd Session of the International Statistical Institute, Bull. Inst. Internat. Statist., vol. 49, 1981, pp. 859-878, 894-902. MR820979

14. J. Segers, Nonparametric inference for bivariate extreme-value copulas, Topics in Extreme Values (M. Ahsanullah and S. N. U. A. Kirmani, eds.), Nova Science Publishers, New York, 2007, 181-203.

15. J. Segers, Asymptotics of empirical copula processes under non-restrictive smoothness assumptions, Bernoulli 18 (2012), no. 3, 764-782. MR2948900

16. J. A. Tawn, Modelling multivariate extreme value distributions, Biometrika 77 (1990), no. 2, $245-253$.

17. A. W. van der Vaart and J. A. Wellner, Weak Convergence and Empirical Processes, Springer, New York, 1996. MR 1385671

18. D. Zhang, M. T. Wells, and L. Peng, Nonparametric estimation of the dependence function for a multivariate extreme value distribution, J. Multivariate Anal. 99 (2008), 577-588. MR2406072

Center of Mathematics of University of Minho, Campus de Gualtar, Braga, Portugal

Current address: CEMAT (Center for Computational and Stochastic Mathematics) of Instituto Superior Técnico, University of Lisbon, Portugal

E-mail address: msferreira@math.uminho.pt

Received 31/JULY/2015

Originally published in English 